\title{
Shifts in apparent spatial frequency induced by metacontrast
}

\author{
CHARLES M. LORBER \\ Macon Junior College, Macon, Georgia 31206 \\ and \\ CHARLES W. WHITE \\ New School for Social Research, New York, New York 10003
}

\begin{abstract}
The apparent spatial frequency of a briefly presented square-wave grating is altered by immediately following it with another briefly presented nonoverlapping square-wave grating. If the second grating is of a higher spatial frequency than the first grating, the first grating appears shifted to a lower spatial frequency. If the second grating is of a lower spatial frequency, the first grating appears shifted to a higher spatial frequency. If the second grating is delayed $75 \mathrm{msec}$, the effect is not observed. The results are compared to similar effects that involve longer term adaptation and overlapping stimuli.
\end{abstract}

An aftereffect of size adaptation has been reported by Blakemore and Sutton (1969). They found that adapting to a grating altered the apparent size of bars in other gratings. In terms of spatial frequency, adapting to a grating caused gratings of slightly higher spatial frequency to appear shifted to higher spatial frequencies; conversely, gratings of slightly lower spatial frequency appeared to shift to lower spatial frequencies. At the same spatial frequency as the adapting grating, no shift in apparent spatial frequency takes place, but the target grating does appear reduced in contrast (Campbell \& Robson, 1968).

Klein, Stromeyer, and Ganz (1974) investigated the different effects of successive adaptation and simultaneous induction on the shift in apparent spatial frequency and threshold detection of target gratings. Their investigation revealed that the apparent shift in spatial frequency occurred in both conditions, while threshold detection was impaired only in the successive condition. White and Lorber (1976) demonstrated spatial frequency specific masking using a metacontrast paradigm. Square-wave gratings of 6 cycles/deg and 12 cycles/deg were used as targets. Nonoverlapping, flanking square-wave gratings of various spatial frequencies were employed as masks. Masking was greatest when the masking grating had approximately the same spatial frequency as the target.

The present study demonstrates that shifts in apparent spatial frequency can also be produced using a metacontrast paradigm with a stimulus configuration similar to the circular target grating and concentric

This research was supported in part by a Biological Sciences support grant to Charles White at Duke University, NIH Grant 5505RR07070-09: Address correspondence to Charles M. Lorber, Division of Social Science, Macon Junior College, Macon, Georgia 31206. annular inducing field used by Klein et al. (1974). This is another indication that transient interactions involving form perception between neighboring retinal areas depend on the spatial frequency components of the forms imaged on these areas.

\section{METHOD}

Subjects

The observers were three male and two female Duke University students with normal or corrected vision who were paid for their participation.

\section{Apparatus}

The stimuli were constructed from matte-black chart tape mounted on white poster board, photographed on high-contrast copy film and printed on semigloss paper. The stimuli were presented in a three-channel tachistoscope (Scientific Prototype, Model GB). One channel contained the comparison stimulus and a fixation point, another channel contained the target, and the third channel contained the inducing field. All stimuli were square-wave gratings, as shown in Figure 1. The target and comparison stimuli were circular fields with a diameter of $1.23 \mathrm{deg}$ visual angle. The inducing field was an annulus with an inner diameter of $1.23 \mathrm{deg}$ visual angle and an outer diameter of $3.70 \mathrm{deg}$ visual angle. The target grating was $4 \mathrm{cycles} / \mathrm{deg}$. The inducing field was either 2 or 8 cycles/deg. Eight comparison stimuli were used, ranging from 3.3 to 4.7 cycles/deg in .2-cycles/deg steps. The mean luminance of each field was approximately $19 \mathrm{~cd} / \mathrm{m}^{2}$. The contrast of each stimulus was .74. Contrast was defined as maximum luminance - minimum luminance/maximum luminance + minimum luminance.

\section{Procedure}

All stimuli were presented monocularly to the right eye. Each observer used a chin and forehead rest to minimize head movements. The stimuli were presented in the following sequence: Before the first presentation, the observer adapted for $2 \mathrm{~min}$ to the comparison field. The observer then viewed the comparison stimulus, located in the left half of the field, shifted his gaze to the fixation point on the right side of the field, and initiated the target-inducing field sequence by pressing a button. The target was presented for $10 \mathrm{msec}$. Following an 


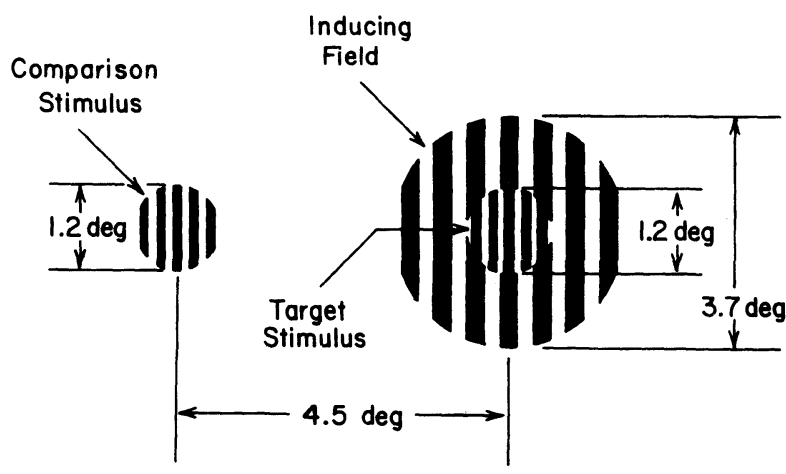

Figure 1. The stimuli as they would appear if presented simultaneously: The comparison stimulus, the target stimulus, the inducing field. The fixation point was centered in the target stimulus.

interstimulus interval (ISI) of either $0 \mathrm{msec}$ or $75 \mathrm{msec}$, the inducing field was either presented for $10 \mathrm{msec}$ or omitted. The observer then examined the comparison stimulus again and reported which stimulus, the target or comparison, appeared to have wider stripes (i.e., a lower spatial frequency). The comparison stimulus was then changed and the sequence was repeated. Five conditions were run: no inducing field, 2-and 8-cycles/deg inducing fields with 0 -msec ISI, and 2-and 8-cycles/deg inducing fields with 75-msec ISI.

The apparent spatial frequency of the target was determined by the method of constant stimuli. For each observer, the number of times each comparison stimulus was judged lower in spatial frequency than the target was converted to a percentage of the 15 times each comparison was presented. These values were then converted to $\mathrm{z}$ scores. The Muller-Urban weights were used to calculate the spatial frequency judged subjectively equal to the target (i.e., the point of subjective equality) for each condition (Woodworth, 1938).

Each series involved one inducing field, one ISI value, and eight comparison stimuli that were presented 15 times in random order. Observers ran one session per day. Each session contained two series separated by a rest period.

\section{RESULTS}

Figure 2 illustrates the results. When the target was followed by an inducing field of lower spatial frequency, the target was judged higher in spatial frequency than when it was followed by an inducing field of higher spatial frequency. The effect was observed only when the inducing field immediately followed the target (i.e., ISI =0).

A one-way repeated-measures analysis of variance produced a significant treatment effect $(p<.025)$, which indicated that the apparent spatial frequency of the target varied as a function of the inducing spatial frequency, or the ISI, or both. A Neuman-Keuls test revealed a significant difference $(p<.05)$ between the high and low spatial frequency inducing field conditions at an ISI of $0 \mathrm{msec}$. None of the other paired comparisons was significant. The apparent spatial frequency of the target depended not only on the presence or absence of an inducing field and the inducing field's spatial frequency, but also the time at which the inducing field was presented.

\section{DISCUSSION}

Klein et al. (1974) found a difference between the induced shift in apparent spatial frequency of a grating and an increase in the grating's contrast threshold, using successive and nonoverlapping simultaneous adaptation procedures. The shift in apparent spatial frequency was induced in both conditions, but contrast threshold increased only in the successive condition, where the same retinal area undergoing adaptation was also required to detect the grating.

The results of a previous experiment showed that gratings could be masked (e.g., reduced in apparent contrast and duration) by gratings of similar spatial frequencies (White \& Lorber, 1976). The results of the present experiment indicate that the apparent spatial frequency of a grating can be shifted by stimulating nonoverlapping retinal areas with other gratings.

Breitmeyer and Ganz (1976) have recently presented a multiple-factor model of visual masking. Their model for metacontrast has the target signal, which is mediated by a sustained system similar to the X-cell system found in cats and some primates, being interrupted by the mask signal, which is carried by a transient system similar to the Y-cell system. They propose that the sustained system is spatial frequency specific, with units responding to higher spatial frequencies having longer latencies. With other parameters held constant, ISI will determine the extent of masking. The mask signal will reach the locations in the visual system where it can interfere with the target signal after a fixed number of milliseconds following presentation. Depending on the ISI, it will interfere with units representing different spatial frequencies. If the ISI is long enough, only high target frequencies would be affected. This might result in a perception of a target that is recognizable, but lacking in sharp contours. A short ISI would theoretically delete low spatial frequencies. The result might be a target that looks like an outline figure (see, e.g., Plate 31 in Harburn, Taylor, \& Welberry, 1975). If some intermediate ISI is used, the mask may block the frequencies that are most important to the particular target and result in the perception of a severely degraded target or no target at all.

The Breitmeyer and Ganz (1976) theory predicts that substituting one target for another (i.e., changing the spatial frequency components) requires only a change in ISI to maintain a constant level of masking. This fails to account for the long line

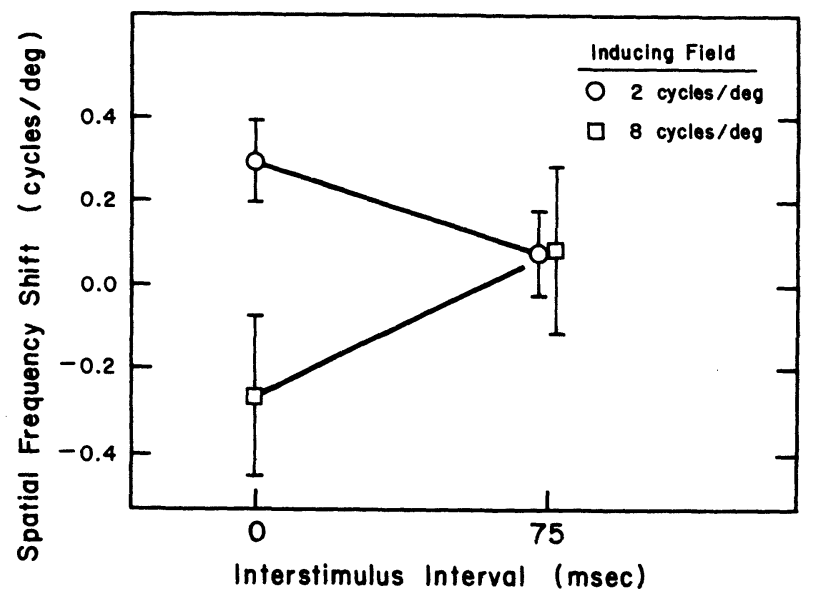

Figure 2. Shifts in apparent spatial frequency. The ordinate indicates the difference in apparent spatial frequency between the target and the comparison stimulus when a 4-cycles/deg target was presented alone and when it was followed by an inducing field of 2 cycles/deg (circles) or 8 cycles/deg (squares). Vertical line segments indicate one standard error above and below each mean. 
of research that has shown metacontrast to be highly form specific (Uttal, 1970, 1971; Werner, 1935). A change in the shape of a target requires a change in the shape of the mask to maintain maximum masking. In the spatial frequency domain, White and Lorber (1976) have shown that metacontrast between nonoverlapping gratings depends on the spatial frequency of the target and mask. The present study lends support to the importance of the mask's spatial frequency content. At a given ISI, masks with different spatial frequencies affected a target differentially.

One way in which the results of the present study may be interpreted using a modified version of the Breitmeyer and Ganz (1976) model is to develop a model along the lines of that proposed by Ganz (1966) to explain figural aftereffects. When a target is presented, units with maximum sensitivity at the spatial frequencies contained in the target are highly stimulated. Units with maximum sensitivities to higher and lower spatial frequencies are stimulated less, with stimulation decreasing with increasing difference in spatial frequency sensitivity. It may be assumed that this unit activity eventually results in the perception of a square-wave grating of a particular spatial frequency. The presentation of the inducing field masks the target stimulus; that is, it may decrease the activity of units on one side of the peak activity. This would result in a shift in the distribution of the responding units in a direction away from the inducing field's spatial frequency. The target, in this case, is perceived as shifted in spatial frequency away from the inducing field's spatial frequency.

We have observed interactions between briefly presented, nonoverlapping grating stimuli that result in a change in the appearance of the stimuli. Frome, Danielson, and Levinson (Note 1) and Frome, Harris, and Levinson (Note 2) have reported long-term changes in two-dimensional stimuli (rectangles) produced by adapting to sine-wave gratings. These studies involved long-term adaptation of the same retinal area used to examine the rectangles. It now appears possible to produce similar changes in two-dimensional stimuli using briefly presented nonoverlapping stimuli. The metacontrast paradigm continues to be a powerful tool for exploring the interactions that occur when neighboring retinal areas are stimulated.

\section{REFERENCE NOTES}

1. Frome, F., Danielson, J. T., \& Levinson, J. Z. Shifts in perception of size after adaptation to gratings. Paper presented at the meeting of the Association for Research in Vision and Opthalmology. Sarasota, Florida, 1974.

2. Frome, F., Harris, C. S., \& Levinson, J. Z. Extremely long-lasting shifts in perception of size after adaptation to gratings. Paper presented at the meeting of the Psychonomic Society. Denver, Colorado, 1975.

\section{REFERENCES}

Blakemore, C., \& Sutton, P. Size adaptation: A new aftereffect. Science, 1969, 166, 245-247.

BreitMeyer, B. G., \& Ganz, L. Implications of sustained and transient channels for theories of visual pattern masking. saccadic suppression, and information processing. Psychological Review, 1976, 83, 1-36.

Campbell, F. W., \& Robson, J. G. Application of Fourier analysis to the visibility of gratings. Journal of Physiology, 1968, 197, 551-556.

Ganz, L. Mechanism of the figural aftereffects. Psychological Review, 1966, 73, 128-150.

Harburn, G., TaYlor, C. A., \& Welberry, T. R. Atlas of Optical Transforms. Ithaca, N.Y: Cornell University Press, 1975.

Klein, S., Stromeyer, C. F., \& Ganz, L. The simultaneous spatial frequency shift: A dissociation between the detection and perception of gratings. Vision Research, 1974, 14, 1421-1432.

UtTal, W. R. On the physiological basis of masking with dotted visual noise. Perception \& Psychophysics, 1970, 7, 321-327.

UTtal, W. R. The psychobiological silly season-or-what happens when neurophysiological data become psychological theories. Journal of General Psychology, 1971, 84, 151-167.

WERNER, H. Studies of contour: I. Qualitative analysis. American Journal of Psychology, 1935, 47, 40-67.

White, C. W., \& LORBER, C. M. Spatial-frequency specificity in visual masking. Perception \& Psychophysics, 1976, 19. 281-284.

Woodworth, R. S. Experimental Psychology. New York: Holt, 1938. 